\title{
LA BIBLIOTECA PÚBLICA Y LAS COMPETENCIAS DEL SIGLO XXI
}

The public library and the skills of the XXI century

Emir-José Suaiden

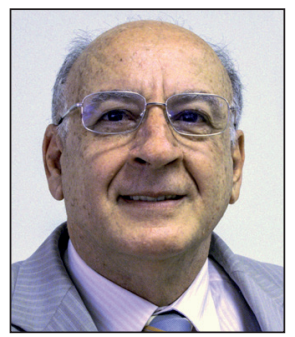

Emir-José Suaiden, profesor titular de la Universidade de Brasilia, es investigador senior del Consejo Nacional de Desarrollo Científico y Tecnológico. En 2012 recibió el Premio de Inclusión Digital de la Telemar, y en 2017 el Premio Rubens Borba de Moraes. Ha dirigido el Instituto Nacional del Libro, el Instituto Brasileño de Información Científica y Tecnológica (Ibict), y el Sistema de Bibliotecas de la Universidad de Brasília. Ha presidido la Fundación de Apoyo a la Investigación, y el Centro de Estudios para el Fomento del Libro Cerlalc/Unesco. Su proyecto Escuela Digital Integrada se ha transformado en Ley siendo de implementación obligatoria en todas las escuelas del Distrito Federal. Son numerosas las citaciones a sus trabajos en las bases de dados de revistas del portal de la Coordenação de Aperfeiçoamento de Pessoal de Nivel Superior (Capes) y en bases abiertas como Live Search. Actualmente es consultor de la Capes, del Consejo Nacional de Desarrollo Científico y Tecnológico $(C N P q)$ y de la Unesco. https://orcid.org/0000-0001-5786-8060

Universidade de Brasilia, Faculdade de Ciência da Informação Campus Universitário Darcy Ribeiro 70910-900 Brasília DF, Brasil emir@unb.br

\section{Resumen}

Desde el inicio del siglo XX la biblioteca pública ha sido muy cuestionada. Inicialmente la crítica mayor estaba centrada en cómo esas bibliotecas, que más parecían depósitos de libros, podrían colaborar al desarrollo de la sociedad industrial y posteriormente postindustrial. Con el advenimiento de la sociedad de la información surgieron nuevos desafíos como la transformación de lo impreso a lo digital, la formación del público lector y la necesidad de tener nuevas competencias en información para superar esos desafíos. Desde sus inicios la biblioteca fue entendida como el templo del conocimiento y de la verdad. En la actualidad los intereses políticos, económicos e ideológicos fomentan la crisis de la post verdad, de la desinformación, de la manipulación de la información y de las fake news. En este contexto la identificación y desarrollo de competencias en información se vuelve esencial y corresponde a la biblioteca pública crear las condiciones para atender las nuevas demandas de la sociedad de la información y del conocimiento. Basándose en estudios comparativos, una revisión bibliográfica y aplicando metodologías cualitativas, este artículo analiza la división de la comunidad, la mediación de la lectura, la experiencia en información, el retorno de la inversión (ROI), el rendimiento social de la inversión (SROI) y cómo el nuevo modelo de biblioteca pública puede hacer frente a las necesidades de los ciudadanos del siglo XXI.

\section{Palabras clave}

Bibliotecas públicas; Competencias; Bibliotecarios; Personal bibliotecario; Público; Lectores; Servicios; Necesidades; Sociedad de la información; Evolución; Desarrollo; Política bibliotecaria.

\begin{abstract}
Since the beginning of the XX century, public libraries have been contested. Initially, the biggest criticism was focused on those libraries which seemed a book deposit, and how they could cooperate to the development of the industrial society and also, afterwards, the post-industrial society. With the coming of the information society, new challenges came up, like the transformation from the print to digital, the training of readers... New skills were demanded to librarians to overcome these challenges. Since their first years of existence, libraries have been understood as temples of knowledge and truth. Nowadays, political, economic and ideological interests support the post-truth crisis, over abundance of information, manipulation of the information and fake news. Within this context, identification and development of information skills has become essential. Moreover, it is a duty of public libraries to create the conditions to satisfy the new demands of the information \& knowledge society. Based on comparative studies, a bibliographic review and qualitative methodologies, this article analyzes the community divide, the reading mediation, the information skills, both the return on investment (ROI) and the social return on investment (SROI), and to demonstrate how the new model of public library is able to cope with the needs of the XXI century's citizens.
\end{abstract}

\section{Keywords}

Public libraries; Competencies; Skills; Librarians; Library personnel; Library staff; Public; Readers; Services; Needs; Information society; Evolution; Development; Library policy. 
Suaiden, Emir-José (2018). "La biblioteca pública y las competencias del siglo XXI". El profesional de la información, v. 27, n. 5, pp. 1136-1144

\section{Introducción}

\subsection{Papel social de la biblioteca pública}

Desde el advenimiento de la sociedad de la información el papel de la biblioteca pública viene, cada vez más, recibiendo críticas por parte de estudiantes, investigadores y profesionales de la información y comunicación. La crítica más dura se refiere a la falta de implementación de un nuevo modelo de biblioteca capaz de hacer frente a las necesidades de información de los ciudadanos del siglo XXI.

Muchos estudios revelan diferencias fundamentales entre las bibliotecas públicas de Europa, principalmente de los Países Bajos, y de los Estados Unidos de Norteamérica cuando se comparan con las bibliotecas de América Latina o de los países africanos.

En los países desarrollados no hay tanta necesidad de concienciar a las autoridades sobre la importancia del papel de la biblioteca pública para la sociedad, pero en los países menos desarrollados la lucha es constante para que los gobiernos las tengan en cuenta. En realidad, el problema se inicia por la falta de sensibilización del público lector. En la mayoría de estos países la biblioteca no forma parte de la agenda gubernamental.

\section{Cuando las bibliotecas no forman parte de la agenda gubernamental no cabe es- perar que reciban recursos financieros}

En América Latina los países que más invierten en bibliotecas son Chile -que tiene la mejor economía de la región y es el país con más patentes registradas-, y Colombia, con su nuevo modelo de biblioteca, titulado Parque Biblioteca, que ha sido fundamental para la disminución de los conflictos en las comunidades menos favorecidas.

Cuando las bibliotecas no forman parte de la agenda gubernamental no cabe esperar que haya presupuesto ni disponibilidad de recursos financieros para ellas. Y por consiguiente no consiguen tener visibilidad ni demostrar la importancia de sus servicios para la sociedad.

En el siglo pasado, instituciones como Unesco, IFLA, OEA, entre otras, lanzaron diversos manifiestos para intentar sensibilizar a las autoridades de América Latina sobre la importancia de las bibliotecas públicas. Fue un esfuerzo con resultados limitados, pues se inauguraron diversas bibliotecas públicas, pero más como depósitos de libros que como instituciones que promueven el libre acceso al conocimiento organizado.

En ese período quedó claro que los anglosajones tenían una mayor capacidad de lectura debido principalmente a su cultura avanzada imbuida en la población y a la importante in- dustria editorial existente en sus países. En América Latina, sin embargo, la formación del público lector dependía de la capacidad del profesional de la información de sensibilizar a las autoridades y a la comunidad sobre la importancia del acceso al libro ya la lectura. El gran problema fue que el currículo de las escuelas de biblioteconomía no privilegiaba la formación y el mantenimiento de público lector: todo el esfuerzo estaba orientado hacia la preservación y no a la divulgación de las colecciones (Bandino, 2000). Si el bibliotecario no tenía capacidad de lectura, poco podía promover y liderar movimientos en pro de la lectura, pues cada vez más se comprueba que sólo un lector puede formar otro lector.

\section{Cada vez más se comprueba que sólo un lector puede formar otro lector}

\subsection{Usuarios de la biblioteca pública}

Otro importante desafío era la cuestión de la biblioteca pública para todos. La falta de recursos financieros adecuados y la diversidad de intereses informacionales de la comunidad demostraron la dificultad de crear bibliotecas para servir a todos indistintamente, por lo que algunas instituciones prefirieron segmentar el mercado dando prioridad a sectores específicos de la comunidad, tales como estudiantes, comerciantes, amas de casa, jubilados, etc.

En ese período, el papel de la biblioteca pública fue dar la palabra al "no público": personas que por analfabetismo, desnutrición infantil y otros problemas no formaban parte del grupo selecto de los ilustrados y por eso no tenían noción de sus derechos y deberes como ciudadanos. Para capacitar a un público como ese, el bibliotecario tenía que haber tenido nociones educativas, sociales y psicológicas. Dar la palabra significa disminuir la distancia entre el libro y el usuario teniendo al bibliotecario como mediador, pues en la medida en que el "no público" tenga acceso a lectura él pasa a tener un discurso crítico propio y aprende a no ser manipulado (Castro; Ribeiro, 1997).

En realidad fueron muchos los problemas a los que se enfrentó la biblioteca pública: falta de concienciación de las autoridades, colecciones obsoletas, falta de recursos humanos, etc. Este clima generó la llamada "escolarización de la biblioteca pública" debido principalmente a la falta de bibliotecas escolares. En la medida en que la prioridad fueron los estudiantes los demás segmentos de la comunidad fueron totalmente olvidados. Priorizando a los estudiantes el acervo básicamente estaba constituido por libros de texto y didácticos, que es reconocido que no forman un público lector.

En Brasil, la falta de visibilidad de la biblioteca pública, el distanciamiento al mundo del libro, la falta de público-lector, la 
elitización de la lectura..., han provocado una serie de problemas que van desde la poca formación ciudadana hasta la cuestión de la producción científica. Se ha comprobado que la mayoría de los estudiantes que ingresaron en las universidades en los últimos tiempos no pasaron por la biblioteca escolar o pública. Son jóvenes que tienen dificultades para interpretar el texto escrito y traen el vicio del corta y pega (Pacheco, 1995). Tienen dificultad para validar la información y no saben agregar valor al texto escrito. La situación se complica cuando plagian para elaborar la monografía, la disertación o la tesis. Es una situación en la que no se promueve el desarrollo y mantiene la dependencia informacional y tecnológica del extranjero. Un indicador importante, que ilustra y corrobora lo aquí planteado, es que Chile superó a Brasil en número de patentes. Eso refleja la calidad de la infraestructura informacional existente que impacta en la calidad de la educación y en el desarrollo.

Dar la palabra significa disminuir la distancia entre el libro y el usuario, teniendo al bibliotecario como mediador

No sólo en Brasil sino en toda América Latina las personas que leen se convierten en lectores críticos, pues según Borges (1962):

"se puede terminar la historia donde se quiera, se puede conectar aquella historia con cualquier otra, se puede negar la historia, olvidar la historia, hacerla un clásico, considerarla sólo un reflejo de otras historias..., todo depende de la capacidad interpretativa del lector".

Cuando los lectores críticos se convierten en usuarios críticos, empiezan a exigir más de los servicios bibliotecarios, y es así como las bibliotecas toman conciencia de la necesidad de mejorar la calidad de los productos y servicios ofrecidos para tales usuarios.

\subsection{Nuevas formas de leer}

En un artículo titulado: "Nuevas trayectorias de lectura", Niskier (2014) afirma que:

"el lector de la Edad Media nació conviviendo con palabras escritas en los carteles, embalajes, placas, revistas, juegos..., la escritura tiene una presencia constante en el mundo".

Según el referido autor, los recursos tecnológicos hoy disponibles permiten que el lector intervenga directamente en los textos, con un mínimo de conocimiento técnico. Además, el hipertexto transfiere parte del poder del escritor al lector, el cual adquiere la posibilidad de elegir libremente sus trayectorias de lectura. Así, el lector elabora lo que podríamos denominar meta-texto, anotando sus escritos junto a escritos de otros autores y estableciendo enlaces (nexos o interconexiones) entre documentos de diferentes autores para relacionarlos y acceder a ellos rápidamente.

En la visión de Pondé (2000), el crecimiento poblacional urbano, las políticas de masificación de la lectura y los diversos modos de reproducción del lenguaje contribuyeron a la ampliación del público lector y el interés por la lectura. EI acto de leer, que en el pasado estaba asociado al respeto por el texto y a la autoridad absoluta del autor, privaba al lector de autoridad, porque presuponía una jerarquía entre el emisor y el destinatario, a medida que se buscaba aquello que el texto o el autor quería decir. La lectura interactiva supone una igualdad entre el texto y el lector en el acto de la comunicación. Con ello, se rompe el esquema autoritario de la relación entre el emisor y el destinatario, y la lectura se transforma en un juego en el cual el lector también asume el papel de autor una vez que da significado al texto y participa activamente de la acción interpretativa. Al reconocer el papel activo del lector, el concepto moderno de autoría queda igualmente comprometido, pues la visión individualista de que sólo el autor es "propietario" del texto se desvanece, ya que la existencia del texto está íntimamente relacionada con su lectura y su interpretación. De esta forma, emisor y receptor tienen autoridad sobre el texto, aunque uno de ellos tiene un poco más de responsabilidad sobre la creación que el otro. Por otra parte, cabe observar que esta concepción burguesa de autoría es reciente, data de mediados del siglo XIX, época en que el proceso de construcción social de la autoría se fortaleció, principalmente debido al desarrollo de la prensa y demás información escrita, y en consecuencia, de las profesiones de periodista y escritor.

Manguel (2002) tiene la visión de que la responsabilidad del escritor debe ser sensible a los descubrimientos de la experiencia; en otras palabras, el escritor debe ser un buen alquimista. La experiencia proporciona material que los escritores transformamos en palabras, pero sólo somos artesanos de esta experiencia. La tarea del autor termina con la construcción de la obra. Cuando la obra está concluida, el escritor no tiene más poder sobre ella, es decir, está en las manos de la persona que la recibe. La responsabilidad del lector es descubrir el texto e intentar hacer un viaje en dirección opuesta para entender la experiencia que sirvió de inspiración. Es posible que el lector descubra otra experiencia, otro mundo a través de esa excursión. El lector tiene una responsabilidad política porque existe un vínculo con la sociedad, con la polis y con lo que Mallarmé ${ }^{1}$ llamó "dar un nuevo significado a las palabras de la tribu".

\section{Los acervos formados exclusivamente por libros de texto y didácticos no ayu- dan a formar un público lector}

\subsection{Impacto social}

¿Y por qué necesitamos lectores críticos? Hay una gran distinción entre el universo de la oralidad y el de la escritura. Desde el punto de vista de reproducción y optimización del sistema la lectura es una necesidad pragmática. El analfabeto es menos productivo y más dependiente, es una forma de inculcación ideológica. El texto consentido y efectivamente promovido es sólo aquel que reproduce valores ideológicos hegemónicos. Aunque se incluya en la "ciudad de las letras", el alfabetizado sigue excluido del "mundo de la escritura", que supone territorios privilegiados caracterizados por formas de discursos y referencias específicas.

La estrecha relación entre lectura y participación social se- 
ría reafirmada por Paulo Freire en su conferencia sobre "la importancia del acto de leer", realizada en el $3^{\text {er }}$ Congresso Brasileiro de Leitura, en 1981 (Freire, 1988). Revisando su trayectoria personal, Freire habló de "palabramundo", de la lectura de mundo que antecede a la lectura de la palabra, para reafirmar la tesis de que,

"en cuanto acto de conocimiento y acto creador, el proceso de alfabetización tiene en el alfabetizando a su sujeto. La lectura crítica de la realidad que se produce en un proceso de alfabetización (asociada sobre todo a ciertas prácticas claramente políticas de movilización y de organización) puede constituirse en un instrumento de acción contra-hegemónica".

Es importante observar que este es el tipo de actividad que llevará a las bibliotecas públicas a convertirse en un verdadero punto de acceso para la sociedad de la información. En Brasil el fenómeno de la escolarización de la biblioteca pública perjudicó mucho la formación de lectores principalmente por la falta de profesionales y de un acervo representativo de literatura infantil y juvenil (Takahashi, 2000).

En realidad, en la medida en que la sociedad de la información es también una sociedad de exclusión, el pasaporte para esa sociedad pasa a ser el capital intelectual, cuyo gran fundamento es la lectura y el hábito de utilizar la información, principales actividades de los servicios bibliotecarios. No estamos hablando del lector ocasional y pasivo ante la falta de calidad de los servicios bibliotecarios, pues las habilidades de leer y escribir no son suficientes para estar apto para responder adecuadamente a las necesidades contemporáneas. Esto nos lleva al concepto que en portugués se llama letramento o literacia que está por encima del concepto de alfabetización. Si un niño sabe leer, pero no puede interpretar lo que lee en un libro, revista o periódico, o si sabe escribir palabras y frases, pero no puede escribir una carta, entonces ese niño está alfabetizado, pero no letrado.

El sistema bibliotecario y el sistema educativo tienen una gran responsabilidad en el proceso de cambio social. El camino analfabeto-alfabetizado-letrado-ciudadano-escritor es extremadamente complejo, pero es la única ruta que llevará al profesional de la información a la satisfacción profesional y, ante todo, representa el camino que conducirá su comunidad a la sociedad de la información.

Para hacer frente a los desafíos de la sociedad del conocimiento la biblioteca tendrá que innovar permanentemente. Y los mayores desafíos son de coordinar la transición de lo impreso a lo digital y, principalmente, construir usuarios productores de información y no más usuarios dependientes de la información. Estos dos factores serán vitales para la construcción de comunidades autosustentables y sociedades más justas.

Las últimas encuestas realizadas en los Estados Unidos de Norteamérica revelan la importancia de la familia en la formación de los hábitos de lectura. Investigaciones recientes revelaron que en las familias donde hay libros los niños se dedican mucho más a los estudios. Por lo tanto en este proceso hay una unión de la familia con la biblioteca y con la calidad de enseñanza ofrecida por la escuela. En algunas escuelas incluso la coordinación pedagógica funciona en la propia biblioteca escolar. Es el primer paso para incluir al individuo en la sociedad de la información (Osakabe, 1982).

\section{El problema}

La falta de visibilidad de la biblioteca pública constituye el mayor problema para su desarrollo. En primer lugar no es visible para las administraciones y con ello no participa de la agenda gubernamental en todos los niveles: nacional, regional y municipal, y sin participación en la agenda los recursos son extremadamente limitados tanto para actualización del acervo como para adquisición de equipamientos y nuevas tecnologías.

\section{La comunidad no presiona a los gobernan- tes para tener más y mejores bibliotecas. Se dice incluso que los políticos no invier- ten en bibliotecas porque no dan votos}

Tampoco existe una interacción adecuada con la comunidad. La comunidad no presiona a los gobernantes para tener más y mejores bibliotecas. Se dice incluso que los políticos no invierten en bibliotecas porque no dan votos. Prácticamente sólo los profesionales de la información tienen la visión de que su institución mejora la calidad de vida de la comunidad, transfiriendo información y conocimiento, formando la ciudadanía y generando empleo y renta (D'Elia, 1980; Madden, 1979).

Otro problema es la propia estructura organizativa de la institución en la formulación de una política bibliotecaria, en la elaboración de diagnósticos de los productos y servicios, en la capacitación de los usuarios y en la construcción de escenarios. La construcción de escenarios es muy valiosa pues muchas veces es necesario abandonar la idea de todo para todos y segmentar para un área que pueda desarrollar aún más la comunidad como es el caso de muchos pequeños municipios que sobreviven básicamente de la agricultura.

Por lo tanto, el problema pasa a ser el siguiente: ¿cómo debe organizarse la biblioteca pública para desarrollar unas competencias en información capaces de atender las necesidades del usuario del siglo XXI, obtener retorno social a las inversiones realizadas, entrar en la agenda del gobierno y generar credibilidad y confianza en la sociedad?

\section{Objetivos}

\subsection{Objetivo general}

Proponer un modelo de biblioteca pública basado en competencia informacional capaz de atender a la sociedad contemporánea.

\subsection{Objetivos específicos}

3.2.1. Identificar competencias informacionales para atender mejor a sus usuarios.

3.2.2. Evaluar los productos y servicios ofrecidos y sus impactos sociales para su mantenimiento, eliminación o necesaria adecuación. 
3.2.3. Proponer metodologías adecuadas para la transferencia de la información.

3.2.4. Desarrollar productos y servicios que reflejen las competencias informacionales identificadas.

3.2.5. Proponer estrategias de marketing para la divulgación de las acciones bibliotecarias.

\section{Contextualización de la sociedad de la información}

Manuel Castells, sociólogo español y profesor de la University of California -junto a Anthony Giddens y a Jürgen Habermas- pertenecen a un grupo de intelectuales contemporáneos que rodean y asesoran a gobernantes en las cuestiones relacionadas con la era informacional y la formación de redes. Al escribir su trilogía, Castells (1999) tuvo como objetivo formular una teoría sistémica de la sociedad de la información capaz de analizar el impacto de las nuevas tecnologías sobre la división del trabajo, la estructura del empleo, el debilitamiento del Estado, los sindicatos y sobre la organización de los medios de comunicación masiva en un mundo globalizado y conectado en redes. Propuso identificar los principales procesos de alcance mundial que transformaron la economía, la cultura y la sociedad en los últimos tiempos, analizando el "cómo" y el "por qué" del actual desmantelamiento del Estado-nación, construido desde la edad moderna, pero también de la crisis de legitimación sufrida por sus instituciones y sus representantes. En este proyecto pretendemos analizar la situación de la biblioteca pública en la crisis de legitimación, pues las estadísticas apuntan que sólo un pequeño porcentaje de la comunidad utiliza sus productos y servicios.

Castells (1990) está convencido de que el final del siglo XX representa no sólo el fin del marxismo, sino también el fin de la "era de la razón". Ambos serían sustituidos por la "era informacional". Para Castells llegó el momento en que deberíamos elaborar nuevos conceptos capaces de expresar los cambios tecnológicos ocurridos en el transcurso de las dos últimas décadas y sintetizarlos en el concepto de "cuarta revolución tecnológica". Esta revolución es fruto de un conjunto de tecnologías convergentes integradas en un bloque: la microelectrónica, la computación (software y hardware), las telecomunicaciones, la electrónica que utiliza la fibra óptica e incluso la bioingeniería genética. Sin embargo, todo esto no habría generado los cambios estructurales de los mercados, de los Estados y del mundo entero si no hubiera habido un feliz matrimonio entre esa tecnología de vanguardia y un mercado ágil y flexible que supo propagar y multiplicar los resultados de la ciencia (nuevos conocimientos) y de esa tecnología, perfeccionada en Silicon Valley, a través de la World Wide Web.

Así, la elaboración de nuevos conceptos, la utilización de las nuevas tecnologías de información y comunicación, y un proceso de cambio enfocado a productos y servicios más atractivos para la sociedad, pasan a ser prioritarios para la visibilidad de la biblioteca pública.

La caracterización de esta revolución tecnológica reciente no es la centralización del saber y de la información (ya presente en las revoluciones anteriores), sino precisamente la comunicación inmediata y fácil de nuevos saberes, de nuevas informaciones y tecnologías al mundo entero a través de redes globales que realimentan e integran nuevas redes, asociadas al mercado y a los poderes económico y político.

\section{La revolución tecnológica sólo está be- neficiando una cuarta parte de la pobla- ción global}

El carácter "excluyente" de este nuevo modo de desarrollo no escapó a Castells: sabe que a lo sumo sólo se está beneficiando una cuarta parte de la población global, pero expresa su esperanza de que tal situación sea sólo temporal. En realidad, las poblaciones más privilegiadas están en los Estados Unidos de Norteamérica, en la Unión Europea en Japón, Corea del Sur y Taiwán. Analizando estos aspectos, un objetivo de la biblioteca pública podría ser hacer frente las desigualdades sociales, diseminando la inclusión informacional productiva que favorece la generación de empleo y renta (Albagli; Maciel; Abdo, 2015; Kliksberg, 1994).

Castells no culpabiliza a la "era informacional" por el desempleo. Por el contrario, afirma que con las nuevas tecnologías se crearon también nuevas formas de empleo. Si se produjeron olas de despidos, ello se debió a la falta de preparación de los trabajadores, ligados a las viejas tecnologías, que no supieron adaptarse a las nuevas condiciones de vida. Así, la capacitación de la mano de obra del usuario de la biblioteca pública pasa a ser prioridad con el fin de hacer frente al período de cambio.

En esta nueva sociedad la ciencia no es vista sólo como una herramienta para aprovechar el conocimiento, sino princi-

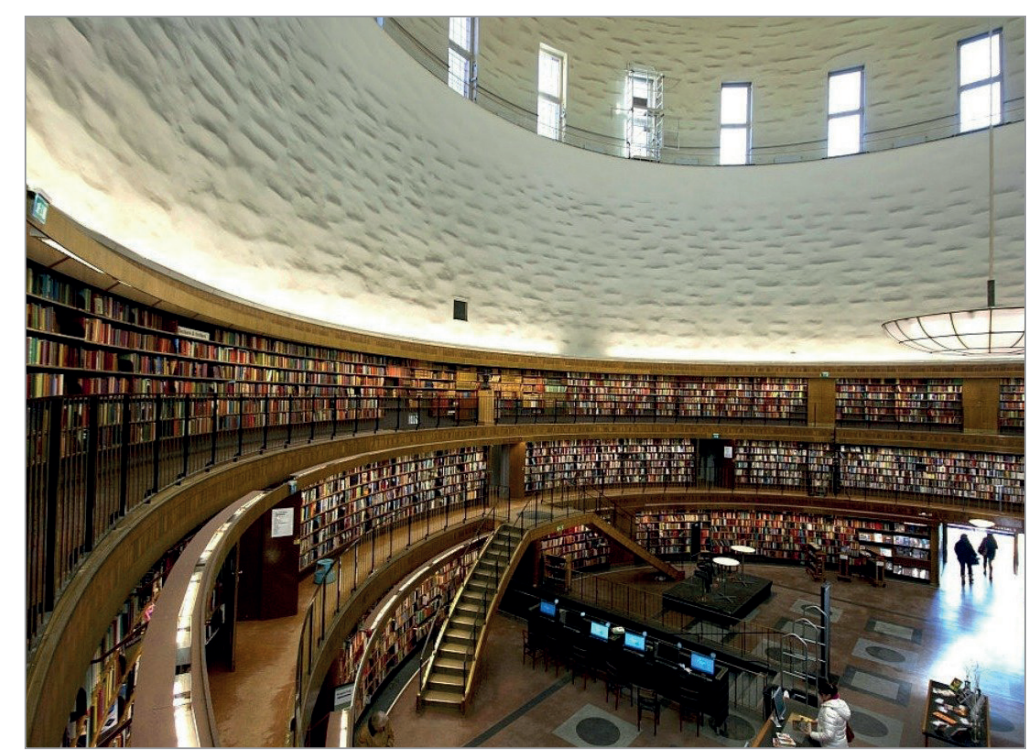

Biblioteca Pública de Estocolmo [Wojtek Gurak/Flickr] https://www.euractiv.com/section/languages-culture/opinion/public-libraries-a-hiddenkey-to-the-success-of-the-digital-single-market 
palmente para mejorar la calidad de vida. En este contexto en el que los desafíos de la sociedad de la información y de las crisis económicas y sociales son gigantescos, la biblioteca pública juega un papel fundamental en la generación de empleo y renta. En Europa, por ejemplo, hay indicadores que confirman que cada año la biblioteca pública encamina al mercado de trabajo más de 250.000 personas. En Rivas Vaciamadrid, por ejemplo, una ciudad española, la biblioteca pública tiene como gran meta la empleabilidad y así promueve actividades de elaboración de currículum vitae, cartas de presentación, y cursos para la formación de mano de obra calificada, además de cursos de idiomas.

http://www.bibliotecaspublicas.es/rivas

\section{La capacitación de la mano de obra del usuario de la biblioteca pública pasa a ser prioridad con el fin de hacer frente al período de cambio}

Lins (2016) realizó un estudio comparativo mostrando como Chile, Colombia y Brasil están en la búsqueda de un nuevo modelo. Chile quiere seguir siendo la mejor economía de la región, Colombia busca un modelo de biblioteca pública que disminuya los conflictos sociales, y Brasil un modelo que incluya a la comunidad en la sociedad de la información.

En realidad, en una sociedad de la información globalizada la biblioteca pública ha enfrentado muchos desafíos y muchas competencias. Será fundamental para el proceso de credibilidad la demostración de que los recursos son bien aplicados y resultará en la mejora de oportunidades para la generación de empleo y renta basadosen la transferencia de la información (Cardoso; De-Oliveira; Fausto, 2018).

\section{Los nuevos retos}

Las bibliotecas públicas implantadas a partir del siglo XVIII principalmente en los países desarrollados, buscaban y dejaron un legado de que el acervo representaba la verdad. Así los usuarios buscaban la comprobación de la verdad a través de las biografías, de los textos seleccionados y de la comprobación científica. Tenían la certeza sobre la vida de los héroes, los mitos y la historia. Las guerras ideológicas poco después de la $2^{\text {a }}$ Guerra Mundial procuraron demostrar que ni siquiera los libros existentes en las bibliotecas eran fuentes confiables para la búsqueda de la verdad. Recientemente el Oxford English Dictionary publicó por primera vez el concepto de pos-verdad, o sea, la verdad ya no tiene tanta consistencia y necesita ser evaluada por otros medios que no sean de interés político o de los medios.

A partir de los noventa comenzaron a surgir publicaciones sobre las teorías de la desinformación. La desinformación atenta los intereses de la hegemonía del poder, pues aumenta el número de países dependientes de todo tipo de comercio. En la mayoría de los países latinoamericanos se utiliza la desinformación para explorar los analfabetos y las personas con desnutrición infantil.

La manipulación de la información es aún más acentuada en esos países donde muchas personas votan por un plato de comida o un subempleo. Son fácilmente manipuladas, pues no tiene noción de ciudadanía y de sus propios derechos.

El Oxford English Dictionary elige a cada vuelta del calendario la palabra del año, la que sobresalió en la comunicación del período. La de 2016 fue fake news -noticias fraudulentas que se multiplican gracias a que se comparten por los medios sociales-. La preferencia de 2017 recayó sobre youthquake, que en traducción libre quiere decir terremoto joven y se trata de un cambio cultural, político o social significativo provocado por las acciones o la influencia de personas jóvenes.

A mediados de 2016 Aviv Ovadya percibió que había algo fundamentalmente equivocado con Internet, tan equivocado que abandonó su trabajo e hizo sonar una alarma. Algunas semanas antes de las elecciones de 2016, presentó sus preocupaciones a tecnólogos en el Área de Bahía de San Francisco y advirtió sobre una crisis inminente de desinformación que llamó infocalypse (Castillo, 2018).

En la visión de expertos estos impactos de desinformación, manipulación, fake news e infocalypse alcanzarán no sólo la política, sino también la gestión, la formación de los usuarios de las bibliotecas, la formación de la ciudadanía, la formación de investigadores y podrán aumentar aún más las desigualdades sociales en América Latina y el Caribe (Kliksberg, 2000). En la medida en que los profesionales de la información luchan por el acceso abierto a la información, por la ciencia abierta, tendrán que afrontar estos nuevos desafíos, que se constituyen en verdaderos obstáculos.

\section{Para comprender el nuevo paradigma}

se hace necesario un pensamiento más amplio, multidimensional, contextualizado y multidisciplinario

Entre las competencias más exigidas para la inclusión en la sociedad de la información está la informacional. Ahí es donde los profesionales de la información podrán capacitar a los usuarios en las investigaciones y estudios sobre la validación de la información.

\section{Procedimientos metodológicos}

Morín (2002) afirma que para comprender el nuevo paradigma (y la biblioteca pública enfrentó el rompimiento de diversos paradigmas tales como el acceso, la comprensión, la validez de la información y el impacto del acceso al libro y la lectura) se hace necesario un pensamiento más amplio, multidimensional, contextualizado y multidisciplinario.

La metodología de investigación utilizada en este estudio privilegió el abordaje cualitativo por ofrecer la facilidad de describir la complejidad de un determinado problema a través de una integración del contexto social y el objeto de estudio. La investigación es también de carácter exploratorio y descriptivo, ya que pretende proporcionar una visión global del fenómeno estudiado. La investigación exploratoria tiene como principal finalidad desarrollar, aclarar y modificar conceptos e ideas, con miras a la formulación de problemas más precisos. Por último, es descriptiva porque tiene como 
objetivo primordial la descripción de las características de determinada población o fenómeno que pretendemos estudiar.

En la primera etapa del trabajo se utilizó la investigación bibliográfica y documental. Esta etapa fue dedicada a la fundamentación teórica proporcionada por los grandes pensadores, tales como Freire, Habermas, Morín, Castells, Levy, Negroponte, Demo, etc. En la cuestión del libro y de la lectura fue fundamental la interpretación de los textos de Roger Chartier, Alberto Manguel, y Paulo Freire. Todo esto se comparó con los textos institucionales publicados por la Unesco, OEA, Cerlalc, Instituto Nacional del Libro, Biblioteca Nacional, Fundación Nacional del Libro Infantil y Juvenil, entre otros. En esta etapa se utilizó la Biblioteca Digital Brasileira de Teses e Dissertações (BDTD) del Ibict, repositorios digitales, bancos y bases de datos y los blogs de diversos intelectuales.

La segunda etapa, ya contando con los fundamentos teóricos, se dedicó a los estudios y técnicas metodológicas. La investigación presenta y traduce la práctica de la observación y del análisis de las dinámicas interactivas y comunicativas. Así, al evaluar programas y proyectos con miras a la recomendación de soluciones a los problemas e impasses identificados se deben tener en cuenta las evidencias de la observación y de la descripción, elementos cruciales de la actividad de investigación.

Las metodologías más importantes que se utilizan son: el retorno a la inversión (return on investment, ROI) y el retorno social a la inversión (SROI). Estas metodologías hoy en día son muy utilizadas en el ámbito anglosajón, fundamentalmente en los Estados Unidos de Norteamérica y en el Reino Unido. En la actualidad, Australia, Nueva Zelanda, Noruega, Corea del Sur y España han utilizado esta metodología, que se aplica principalmente a organizaciones sin fines de lucro para demostrar el impacto social y económico de sus actividades en: generación de empleo, economía en los servicios de salud, proceso de ciudadanía, e incluso en la mejora y visibilidad de la producción científica. Los indicadores demuestran que la biblioteca pública debe participar cada vez más en la agenda del gobierno. Los economistas cada vez más comprueban que un buen sistema educativo y cultural tiene impacto directo en el crecimiento del PIB (producto interno bruto).

Una de las etapas importantes del proyecto es identificar las buenas prácticas a través de estudios comparativos. Por ejemplo, Guadalajara es la ciudad con más lectores según los editores españoles, gracias al trabajo de la biblioteca pública. Colombia implantó Parques Biblioteca, en lugar de la biblioteca pública tradicional, y con ello formó un público lector mucho más consistente. Hoy es posible reunir en una videoconferencia a los responsables de esos cambios que fueron importantes en la visibilización de la biblioteca pública, en la formación del público lector y en la inserción social. En este contexto las técnicas de la narrativa (Brusamolin;
Suaiden, 2014) son importantes en el proceso de gestión y diseminación de la información.

Los grandes cambios están ocurriendo en el propio concepto y en el proceso de integración con la comunidad. Nuevas bibliotecas públicas están utilizando las técnicas de segmentación de mercado (Kotler, 2017) también llamadas técnicas de segmentación de la comunidad. En este proceso la biblioteca pública deja de ser "todo para todos" y atiende prioritariamente, con eficacia y eficiencia, un sector específico con información para generar empleo y renta, o información para el desarrollo del agronegocio, o información para la consolidación del público lector, o para que los estudiantes ingresen en la universidad.

También son fundamentales las técnicas de mediación de la lectura, que tienen como principal objetivo las prácticas de aprendizaje informacional que al final del proceso posibilita que el estudiante deje de copiar y pase a ser autor. Es importante que deje de ser dependiente y se convierta en productor de información.

\section{Consideraciones}

Los expertos comprueban que hay una gran insatisfacción en la sociedad con respecto a los políticos, su gestión, sus desmanes y con el crecimiento de la corrupción. La historia comprueba que en esa situación la sociedad se revuelve y busca otros caminos más solidarios y compatibles con el desarrollo humano. Fue así en mayo de 1968 en Francia donde el lema: "está prohibido prohibir" mostraba que los jóvenes nacidos después de la $2^{\text {a }}$ Guerra Mundial clamaban por una sociedad más justa e igualitaria. La historia demuestra también que la difusión de la información a todos los niveles se enfrenta a grandes desafíos al encontrarse con la innovación. Fue así cuando Gutenberg inventó la imprenta, trayendo nuevos métodos de reproducción del conocimiento, pero trayendo al mismo tiempo las cuestiones del analfabetismo y de la necesidad de la inclusión educativa (Barreto, 1994). La revolución tecnológica aporta innumerables beneficios, 
como la preservación digital de las revistas electrónicas, de las bases de datos, pero también ha colaborado a aumentar las desigualdades sociales ocasionadas por la exclusión digital. Son nuevos desafíos que la biblioteca pública debe afrontar para diseminar la información y el conocimiento.

En una sociedad de la información / conocimiento donde la verdad es a menudo cuestionada por los dueños del poder, por la hegemonía existente, por los intereses contrariados, por las ideologías y las políticas implementadas, la biblioteca debería ser un centro de resistencia en busca de la verdad. En una sociedad soñadora y romántica es posible imaginar una biblioteca pública ofreciendo indicadores contra la corrupción, contra los desmanes gubernamentales, contra la injusticia y los desequilibrios sociales, y demostrando que el alto importe de los recursos invertidos en la corrupción podría ser utilizado para una educación de calidad, principalmente en la formación de un público lector con competencias para validar la información.

\section{La biblioteca debería ser un centro de resistencia en busca de la verdad}

Todo esto ocurre porque en la actualidad hay una separación entre la sociedad y la política, y el ciudadano no se siente representado por los políticos. Los conceptos utilizados por Manuel Castells, Anthony Giddens y Ulrich Beck aseguran que las personas tienden a ser más inteligentes, rebeldes y creativas que en el pasado, donde había mayor conformismo con un destino preestablecido. Hoy en día cuando son llamadas a hacer juicio de valor y a tomar decisiones en la vida personal y profesional, el comportamiento es otro, bastante diferente de antes en la mayoría de los casos.

Si las nuevas tecnologías, por un lado, extienden las posibilidades de comunicación e información entre los ciudadanos, por otro amplían el impacto de las técnicas y estrategias de manipulación de masas. En los medios sociales actúan robots que estimulan polarizaciones e estorban consensos y acuerdos. Asistimos también en los últimos años a un avance impresionante del corporativismo. En muchas bibliotecas universitarias son los funcionarios los que toman las decisiones de cierre, de adquisiciones, de prioridades presupuestarias..., cuando, en realidad estas decisiones deberían ser tomadas por los usuarios.

En el proceso de capacitar al usuario en la comprensión de la información, la bibliografía investigada apunta diversos caminos sobre métodos de detección automática e información de red (metadatos), pero en la actual situación de América Latina el mejor modelo ha sido la competencia o la alfabetización informacional enfocada al aprendizaje continuo.

En la búsqueda de esas competencias la biblioteca tiene que confiar en que el usuario dependiente de hoy puede transformarse en el investigador de mañana. Como usuario dependiente pasó por una inclusión precaria, como millones de latinoamericanos, aprendiendo a investigar copiando de diccionarios y enciclopedias y posteriormente copiando de la internet. En un contexto de copia y pega el usuario jamás llegará a ser autor.

\section{El usuario dependiente de hoy puede transformarse en el investigador de ma- ñana}

Probablemente hoy no basta tener el conocimiento organizado. La organización del conocimiento es importante, pero tiene poco interés para el usuario que en general utiliza Google cuando necesita información. Según las investigaciones realizadas en ese proyecto, el nuevo modelo de biblioteca pública además de poner a disposición de los lectores acervo bibliográfico tiene que transformarse en un centro de capacitación. En este centro de capacitación la prioridad es la competencia informacional donde los profesionales de la información, principalmente del sector de referencia, pasan a dar cursos frecuentes sobre normas técnicas, citas, investigación en grupo, referencias bibliográficas.... Deben enseñarse los caminos para evitar el plagio y profundizar los conocimientos para la formación del investigador, tales como la producción científica, la internacionalización y la construcción de la visibilidad. Por encima de todo colaborar en la formación de lectores críticos, que no dependan del texto escrito, sino que colaboren agregando valor. En el proceso de validación de la verdad el usuario tendrá condiciones de buscar siempre con autonomía el mejor camino a seguir.

\section{Nota}

1. Stéphane Mallarmé, cuyo verdadero nombre era Étienne Mallarmé (1842-1898) fue un poeta y crítico literario francés.

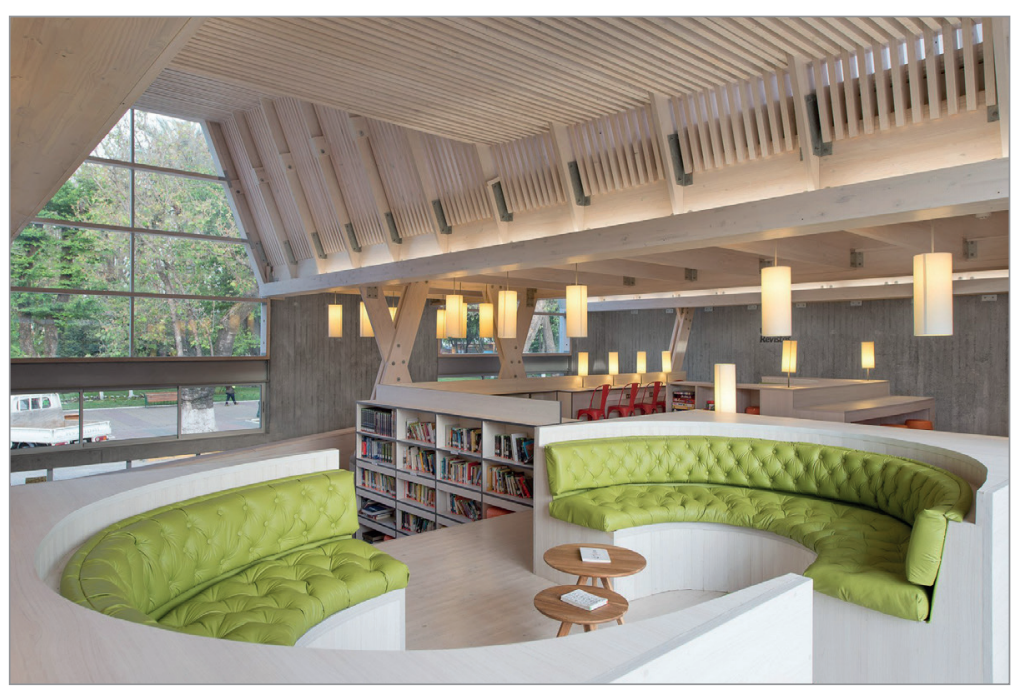

Biblioteca Pública de Constitución, Chile http://www.fundacionlafuente.cl/biblioteca-publica-de-constitucion-es-galardonadapor-la-universidad-mayor 


\section{Referencias}

Albagli, Sarita; Maciel, Maria-Lucia; Abdo, Alexandre-Hannud (2015). Ciência aberta, questões abertas. Brasília, Ibict, Rio de Janeiro, Unirio.

http://livroaberto.ibict.br/bitstream/1/1060/1/Ciencia\%20 aberta_questoes\%20abertas_PORTUGUES_DIGITAL\%20 \%285\%29.pdf

Bandino, Simona (2000). "Prefacio". In: El público y la biblioteca: metodologías para la difusión de la lectura. Gijón: Ediciones Trea, 2000. ISBN: 8495178842

Barreto, Aldo-de-Albuquerque (1994). "A questão da informação". São Paulo em perspectiva, São Paulo, v. 8, n. 4, pp. 3-8.

http://bogliolo.eci.ufmg.br/downloads/BARRETO\%20A\%20 Questao\%20da\%20Informacao.pdf

Borges, José-Luis (1962). The library of Babel. Labyrinths: Selected stories and other writings. Penguin, Harmondsworth. ISBN: 9780811216999

Brusamolin, Valério; Suaiden, Emir-José (2014). Aprendizagem organizacional: o impacto das narrativas. Editora Appris. ISBN: 8581924646

Cardoso, Fernando-Henrique; De-Oliveira, Miguel-Darcy; Fausto, Sergio (2018). Crise e reinvenção da politica no Brasil. São Paulo, Companhia das Letras. ISBN: 9788535930986

Castillo, Toni (2018). "El tecnólogo que predijo las 'fake news' cree que lo siguiente será un apocalipsis informativo". Genbeta, 15 febrero.

https://www.genbeta.com/seguridad/el-tecnologo-quepredijo-las-fake-news-cree-que-lo-siguiente-sera-unapocalipsis-informativo

Castro, César-Augusto; Ribeiro, Maria-Solange-Pereira (1997). "Sociedade da informação: dilema para o bibliotecário". Transinformação, v. 9, n. 1, pp. 17-25.

https://seer.sis.puc-campinas.edu.br/seer/index.php/ transinfo/article/download/1589/1561

D’Elia, George (1980). "Development and testing of a conceptual model of public library user behavior". The library quarterly, v. 50, n. 4, pp. 410-430.

https://www.journals.uchicago.edu/doi/abs/10.1086/601016 https://doi.org/10.1086/601016

Freire, Paulo (1988). A importância do ato de ler: em três artigos que se completam. Cortez Editora. ISBN: 97885 24916465

http://www.paulofreirebymateusbadan.xpg.com.br/Livro2.PDF http://perio.un/p.edu.ar/catedras/system/files/la importancia_del_acto_de_leer.pdf

Kliksberg, Bernardo (1994). Pobreza, uma questão inadiável: novas respostas a nivel mundial. Brasília, DF: Fundação
Escola Nacional de Administração Pública; Enap. ISBN: 978 9806125223

Kliksberg, Bernardo (2000). Desigualdade na América Latina - o dialogo adiado. São Paulo. Cortez Editora.

http://unesdoc. unesco.org/images/0013/001308/130860PORb. $p d f$

Kotler, Philip (2017). My adventures in marketing: The autobiography of Philip Kotler. Idea Bite Press. ISBN: 9780 990576778

Lins, Ivana-Aparecida-Borges (2016). Biblioteca pública, convergências e divergências: Chile, Colômbia e Brasil. Salvador, 198 pp. Tese (doutorado em Ciência da Informação), Programa de pós-graduação em Ciência da Informação-PPGCl/Universidade Federal da Bahia-UFBA, Orientadora: De-Carvalho, Kátia. Resumen:

https://doi.org/10.9771/rpa.v11i2.24917

https://portalseer.ufba.br/index.php/revistaici/article/ view $/ 24917$

Madden, Michael (1979). "Lifestyles of library users and nonusers". Occasional paper n. 137, University of Illinois Graduate School of Library Science, 1-73.

https://www.ideals.illinois.edu/bitstream/handle/2142/3950/ gslisoccasionalpv00000i00137.pdf

Manguel, Alberto (2002). "La lectura electrónica es casi contraria a la lectura misma. Entrevista”. Javier Rodríguez-Marcos. El país, 10 enero.

https://bit.ly/2MB8Qp3

Morín, Edgar (2002). Introducción a una política del hombre. Barcelona: Gedisa. ISBN: 9788474329025

Niskier, Arnaldo (2014). "Novos trajetos de leitura". Correio Braziliense, Brasília, DF, 29 mar. 2014.

http://www.academia.org.br/artigos/novos-trajetos-deleitura

Osakabe, Haquira (1982). "Considerações em torno do acesso ao mundo da escrita". In: Regina Zilberman (org.). Leitura em crise na escola: as alternativas do professor. Porto Alegre, Mercado Aberto.

Pacheco, Leila-Maria-Serafim (1995). "Informação enquanto artefato". Informare: Cadernos do Programa de Pós-graduação em Ciência da Informação, Rio de Janeiro, v. 1, n. 1, pp. 20-24.

http://www.brapci.inf.br/index.php/article/view/0000003099

Pondé, Glória-Maria-Fialho (2000). Leitura e cidadania. Proler, Rio de Janeiro: Biblioteca Nacional.

Takahashi, Tadao (2000). Sociedade da informação no Brasil. Livro verde. Programa Sociedade da Informação (SocInfo).

https://www.governodigital.gov.br/documentos-earquivos/livroverde.pdf 